PROCEEDINGS OF THE

AMERICAN MATHEMATICAL SOCIETY

Volume 127, Number 2, February 1999, Pages 425-435

S 0002-9939(99)04532-3

\title{
RESOLUTION OF SINGULARITIES OF CONVOLUTIONS WITH THE GAUSSIAN KERNEL
}

\author{
KATHRIN BERKNER
}

(Communicated by Frederick W. Gehring)

\begin{abstract}
We present a complete classification of the zero set of a function which is a convolution with the Gaussian kernel. In the first part, we calculate the Taylor expansion of the convolution in a critical point. In the second part, we resolve the singularity with the help of the general Newton process which yields the Puiseux expansions for the solutions. Finally, we describe the resolved singularity in terms of Hermite polynomials.
\end{abstract}

\section{INTRODUCTION}

We consider the zero set of the function $T_{\theta}[f]: \mathbb{R} \times \mathbb{R}_{+} \rightarrow \mathbb{R}$ which is given by the following convolution integral:

$$
T_{\theta}[f](b, a)=\frac{1}{a} \int_{-\infty}^{\infty} \theta\left(\frac{x-b}{a}\right) f(x) d x,
$$

where $f \in L^{2}(\mathbb{R})$ and $\theta(x)=1 / \sqrt{2 \pi} \exp \left(-\frac{1}{2} x^{2}\right)$ denotes the Gaussian function. We call the function $T_{\theta}[f]$ a Gauss convolution (because $\theta$ is is a symmetric function, the function $T_{\theta}[f]$ satisfies the standard definition of a convolution). In a regular point of the zero set of $T_{\theta}[f]$, i.e. $\frac{\partial T_{\theta}[f]}{\partial b}\left(b_{0}, a_{0}\right) \neq 0$ or $\frac{\partial T_{\theta}[f]}{\partial a}\left(b_{0}, a_{0}\right) \neq 0$, the implicit function theorem gives a description of the solution of $T_{\theta}[f]=0$ in a neighborhood of $\left(b_{0}, a_{0}\right)$ as a smooth curve. This case has attracted a lot of interest in the field of signal processing and has been studied by several authors $([1,2,3,9,10])$. In [7], the zero set of $T_{\theta}[f]$ near a critical point is studied with restrictions on $f$ (periodic and bandlimited) in filter theory and its applications.

Even though the classification problem of zeros of $T_{\theta}[f]$ arises from applications in signal processing, it is located in the field of classical real analysis. The analysis of zeros of $T_{\theta}[f]$ that are critical points is the contents of this paper. A complete classification is given by the following main theorem:

Theorem 1.1. Let $f \in L_{\theta}^{2}(\mathbb{R})=\left\{g: \mathbb{R} \rightarrow \mathbb{R}: \int g^{2}(x) e^{-\frac{1}{2} x^{2}} d x<\infty\right\}$ and $\left(b_{0}, a_{0}\right) \in \mathbb{R} \times \mathbb{R}_{+}$with $T_{\theta}[f]\left(b_{0}, a_{0}\right)=0$.

Received by the editors May 15, 1997.

1991 Mathematics Subject Classification. Primary 32S45; Secondary 44A35.

Key words and phrases. Resolution of singularities, convolutions, bifurcations, orthogonal polynomials.

Supported in part by Texas ATP under grant number TATP 003604-018 and by Alexander von Humboldt-Stiftung, Germany.

(C)1999 American Mathematical Society 
1) If $\frac{\partial T_{\theta}[f]}{\partial b}\left(b_{0}, a_{0}\right) \neq 0$, then there exist neighborhoods $U$ of $b_{0}$ and $V$ of $a_{0}$ such that there is a unique differentiable function $h: V \rightarrow U$ with $h\left(a_{0}\right)=b_{0}$ and $T_{\theta}[f](h(a), a)=0$. The solutions $(h(a), a)$ are the only zeros of $T_{\theta}[f]$ in $U \times V$.

2) If there exists an $n>1, n=2 p$ or $n=2 p+1$, such that $\frac{\partial^{m} T_{\theta}[f]}{\partial b^{m}}\left(b_{0}, a_{0}\right)=0$ for all $m<n$ and $\frac{\partial^{n} T_{\theta}[f]}{\partial b^{n}}\left(b_{0}, a_{0}\right) \neq 0$, then there are neighborhoods $U$ of $b_{0}$ and $V$ of $a_{0}$, real numbers $t_{j}<0, t_{i} \neq t_{j}$ and $n_{j} \in \mathbb{N}$ for $j=1, \ldots p$ and $p$ convergent power series $h_{j}: U \rightarrow V$ with

$$
h_{j}(b)=a_{0}+t_{j}\left(b-b_{0}\right)^{2}+\sum_{i=1}^{\infty} a_{i, j}\left(b-b_{0}\right)^{2+\frac{i}{n_{j}}},
$$

such that $T_{\theta}[f](b, a) b=0$.

(a) Let $n=2 p$ and let $x_{1}, \ldots, x_{p}$ denote the $p$ positive roots of the Hermite polynomial of order $2 p$. Then $t_{j}=-\frac{1}{2 a_{0} x_{j}^{2}}$ and the elements of

$$
\bigcup_{j=1}^{p}\left\{\left(b, h_{j}(b)\right) \mid b \in U\right\}
$$

are the only zeros of $T_{\theta}[f]$ in $U \times V$.

(b) Let $n=2 p+1$ and let $y_{1}, \ldots, y_{p}$ denote the $p$ positive roots of the Hermite polynomial of order $2 p+1$. Then $t_{j}=-\frac{1}{2 a_{0} y_{j}^{2}}$ and the elements of

$$
\left\{\left(b_{0}, a\right) \mid a \in V\right\} \quad \cup \bigcup_{j=1}^{p}\left\{\left(b, h_{j}(b)\right) \mid b \in U\right\}
$$

are the only zeros of $T_{\theta}[f]$ in $U \times V$.

3) If $\frac{\partial^{n} T_{\theta}[f]}{\partial b^{n}}\left(b_{0}, a_{0}\right)=0$ for all $n \in \mathbb{N}$, then $f \equiv 0$ almost everywhere.

In the following, we will present a complete proof of the above classification theorem. In section 2, we begin with a summary of results on convolutions with the Gaussian kernel and its relations to the Hermite polynomials. In section 3, we collect all necessary facts on the theory of Puiseux series which provides us with a tool to resolve singularities. Section 4 contains the proof of Theorem 1.1. Finally, in section 5 , we characterize piecewise $\mathcal{C}^{1}$-functions $f: \mathbb{R} \rightarrow \mathbb{R}$, such that the zero set of $T_{\theta}[f]$ has a singularity of a given type.

\section{Convolutions with the Gaussian Kernel}

The following equation for the partial derivatives of $T_{\theta}[f]$ with respect to $b$ follows easily from interchanging differentiation and integration:

$$
\frac{\partial^{n} T_{\theta}[f]}{\partial b^{n}}(b, a)=\frac{(-1)^{n}}{a^{n+1}} \int_{-\infty}^{\infty} \theta^{(n)}\left(\frac{x-b}{a}\right) f(x) d x .
$$

Moreover, the derivatives of the Gaussian function satisfy the following recursion formula:

$$
\theta^{(n)}(x)=-\left[(n-1) \theta^{(n-2)}(x)+x \theta^{(n-1)}(x)\right] .
$$

This relation yields expressions for the partial derivatives of $T_{\theta}[f]$ with respect to $a$. 
Lemma 2.1. For each $n \in \mathbb{N}$, there are constants $c_{i, n} \in \mathbb{R}$ for $i=1, \ldots n$, such that

$$
\frac{\partial^{n} T_{\theta}[f]}{\partial a^{n}}(b, a)=\frac{1}{a^{n+1}} \sum_{i=1}^{n} c_{i, n} \int_{-\infty}^{\infty} \theta^{(2 i)}\left(\frac{x-b}{a}\right) f(x) d x .
$$

Moreover, $c_{n, n}=1$ for each $n>0$.

Proof. Equation (4) is proved by induction over $n$, using the recursion formula (3).

Corollary 2.2. Let $\left(b_{0}, a_{0}\right) \in \mathbb{R} \times \mathbb{R}_{+}$and $n \in \mathbb{N}$ such that

$$
\frac{\partial^{m} T_{\theta}[f]}{\partial b^{m}}\left(b_{0}, a_{0}\right)=0 \text { for all } m<n \text { and } \frac{\partial^{n} T_{\theta}[f]}{\partial b^{n}}\left(b_{0}, a_{0}\right) \neq 0 \text {. }
$$

Then

$$
\frac{\partial^{i+k} T_{\theta}[f]}{\partial a^{i} b^{k}}\left(b_{0}, a_{0}\right)=\left\{\begin{array}{cl}
\left(a_{0}\right)^{i} \frac{\partial^{n} T_{\theta}[f]}{\partial b^{n}}\left(b_{0}, a_{0}\right) & \text { for all } i, k \in \mathbb{N}, \text { where } 2 i+k=n, \\
0 & \text { for all } i, k \in \mathbb{N}, \text { where } 2 i+k<n
\end{array}\right.
$$

The proof is a consequence of Eq.(2) and Lemma 2.1.

The Gaussian function and its derivatives are closely related to Hermite polynomials. These polynomials will play an important role in the following parts of this paper. Therefore, we summarize a few of their properties. For further reading we refer to [5] and [6].

We start with one possible definition. The Hermite-Polynomial $H_{n}(x)$ of order $n$ is defined by

$$
H_{n}(x)=(-1)^{n} e^{\frac{1}{2} x^{2}} \cdot \frac{d^{n}}{d x^{n}}\left(e^{-\frac{1}{2} x^{2}}\right) .
$$

Our definition differs slightly from the definition given in [5] and [6]. It will turn out that our definition is more convenient for the further application to the Gaussian function. Nevertheless, the main properties of Hermite polynomials remain true for our definition.

Polynomial expansion:

$$
H_{n}(x)=x^{n}-\left(\begin{array}{l}
n \\
2
\end{array}\right) x^{n-2}+1 \cdot 3\left(\begin{array}{c}
n \\
4
\end{array}\right) x^{n-4}-1 \cdot 3 \cdot 5\left(\begin{array}{l}
n \\
6
\end{array}\right) x^{n-6}+\ldots .
$$

Generating function:

$$
e^{t x-\frac{1}{2} t^{2}}=\sum_{n=0}^{\infty} H_{n}(x) \frac{t^{n}}{n !}
$$

Orthogonality property:

$$
\int_{-\infty}^{\infty} H_{n}(x) H_{m}(x) e^{-\frac{1}{2} x^{2}} d x=\delta_{n, m} n ! \sqrt{2 \pi} .
$$

If we define $r(x)=e^{-\frac{1}{2} x^{2}}$, then the Hermite polynomials form a complete orthogonal system with respect to the scalar product $\langle f, g\rangle_{r}=\int f(x) g(x) r(x) d x$ for 
$f, g \in L_{\theta}^{2}(\mathbb{R})$. In this notation, we have

$$
\frac{\partial^{n} T_{\theta}[f]}{\partial b^{n}}(b, a)=\frac{1}{a^{n}}\left\langle H_{n}, f(a \cdot+b)\right\rangle_{r} .
$$

In the following, we are interested in the zeros of $T_{\theta}[f]$. In order to characterize the possible shapes of the zero set, we consider the Taylor expansion of $T_{\theta}[f]$ in a point $\left(b_{0}, a_{0}\right)$ with $T_{\theta}[f]\left(b_{0}, a_{0}\right)=0$ :

$$
T_{\theta}[f](b, a)=\sum_{i, k=0}^{\infty} \frac{1}{k ! i !}\left(b-b_{0}\right)^{k}\left(a-a_{0}\right)^{i} \frac{\partial^{k+i} T_{\theta}[f]}{\partial a^{i} \partial b^{k}}\left(b_{0}, a_{0}\right) .
$$

In the next section, we will show how certain conditions on the coefficients of the Taylor expansion allow us to resolve a critical point of the zero set.

\section{Resolution of Singularities with Puiseux series}

Usually, there is no general method to classify the set of zeros of a two-dimensional function $w: \mathbb{R}^{2} \rightarrow \mathbb{R}, w \in \mathcal{C}^{\infty}\left(\mathbb{R}^{2}\right)$, in a neighborhood of a singular point, even if $w$ is represented by its infinite Taylor expansion. If we know more about the partial derivatives of $w$ in a singular point, it is sometimes possible to reduce the Taylor expansion to a form which allows a resolution of the singularity. One of the well known methods to resolve singularities is the Newton Process, see e.g. [4], which we describe next.

Let $w: \mathbb{R}^{2} \rightarrow \mathbb{R}$ be in $\mathcal{C}^{\infty}\left(\mathbb{R}^{2}\right)$ given by the convergent power series

$$
w(x, y)=\sum \beta_{k, i} x^{k} y^{i}
$$

with $\beta_{k, i} \in \mathbb{R}$ and $w(0,0)=0$ near $(0,0)$. Under certain conditions on the coefficients $\beta_{k, i}$, we can find parametrizations of all solutions of $w(x, y)=0$. Here we will briefly explain the main steps of this process.

Without loss of generality let $m>0, \beta_{0, m} \neq 0$ and $\beta_{i, m}=0 \forall i<m$. Furthermore, let $k_{0}>0, i_{0}>0$ with $\beta_{k_{0}, i_{0}} \neq 0$. If there are positive rational numbers $\mu_{0}$ and $\nu$ with $\mu_{0} m=\nu$ such that

$$
w(x, y)=\sum_{k+\mu_{0} i \geq \nu} \beta_{k, i} x^{k} y^{i}
$$

we can split $w$ into a sum of a polynomial $\tilde{w}$ and a remainder $h$ :

$$
\begin{aligned}
\tilde{w}(x, y) & =\sum_{k+\mu_{0} i=\nu} \beta_{k, i} x^{k} y^{i}, \\
h(x, y) & =\sum_{k+\mu_{0} i>\nu} \beta_{k, i} x^{k} y^{i} .
\end{aligned}
$$

With the substitution $y=t x^{\mu_{0}}$ for $t \in \mathbb{R}$ we obtain

$$
\tilde{w}(x, y)=\tilde{w}\left(x, t x^{\mu_{0}}\right)=x^{\nu} \sum_{k+\mu_{0} i=\nu} \beta_{k, i} t^{i}=x^{\nu} g(t),
$$

where $g$ is a polynomial in $t$ of order $m$. If $g$ has a real root $t_{0} \neq 0, y_{0}=t_{0} x^{\mu_{0}}$ is a solution of $\tilde{w}\left(x, y_{0}\right)=0$ and a first approximation to the zeros of $f$. Since $\mu_{0} \in \mathbb{Q}_{>0}$ there are $p_{0}, q_{0} \in \mathbb{N}$ with $\mu_{0}=\frac{p_{0}}{q_{0}}$ and $\left(p_{0}, q_{0}\right)=1$. The substitution $x_{1}=x^{\frac{1}{q_{0}}}$ and $y=x_{1}^{p_{0}}\left(t_{0}+y_{1}\right)$ leads to a new power series $w_{1}$ in $x_{1}$ and $y_{1}$ with

$$
w\left(x_{1}, x_{1}^{p_{0}}\left(t_{0}+y_{1}\right)\right)=x_{1}^{\nu q_{0}} w_{1}\left(x_{1}, y_{1}\right) .
$$


The iterative substitution process $y_{i}=x_{i}^{\mu_{i}}\left(t_{i}+y_{i+1}\right)$ with $\mu_{i}=\frac{p_{i}}{q_{i}} \in \mathbb{Q}(i=0,1, \ldots)$ gives the solution

$$
y=t_{0} x^{\mu_{0}}+t_{1} x^{\mu_{0}+\frac{\mu_{1}}{q_{0}}}+t_{2} x^{\mu_{0}+\frac{\mu_{1}}{q_{0}}+\frac{\mu_{2}}{q_{0} q_{1}}}+\ldots .
$$

In [4] it is proved that there is an index $i_{0}$, such that $\mu_{1} \in \mathbb{N}$ for all $i \geq i_{0}$. Hence $y$ is presented as a power series in $x^{\frac{1}{n}}$ where $n=q_{0} q_{1} \ldots q_{i_{0}}$. The power series

$$
y(x)=\sum_{i=n \mu_{0}} \alpha_{i}\left(x^{\frac{1}{n}}\right)^{i}
$$

is called Puiseux expansion for the curve with equation $f(x, y)=0$. The convergence of the Puiseux expansion follows from the Weierstrass preparation theorem (see e.g. [4]). It is important to mention that we get the complete zero set of $f$ if we determine the Puiseux expansions for all real roots of the polynomial $g$.

\section{Proof of Theorem 1.1}

In this section we present the proof of the Main Theorem 1.1 stated in the indroduction.

Ad 1) This is a consequence of the implicit function theorem.

Ad 3) By Equation (6), the condition $\frac{\partial^{n} T_{\theta}[f]}{\partial b^{n}}\left(b_{0}, a_{0}\right)=0$ for all $n \in \mathbb{N}$ can be rewritten as $\left\langle H_{n}, f(a \cdot+b)\right\rangle_{r}=0$ for all $n \in \mathbb{N}$. Since $\left\{H_{n}\right\}$ is complete, it follows that $f\left(a_{0} x+b_{0}\right)$ is orthogonal to all Hermite polynomials, which implies that $f$ is equal to zero except on a set of measure zero.

Ad 2) We will start with some necessary lemmata.

Lemma 4.1. Let $k \in \mathbb{N}$, and let

$$
P_{k}(x, y)=\frac{1}{k !} y^{k}+\frac{1}{(k-1) ! 2 !} x^{2} y^{k-1}+\ldots+\frac{1}{(2 k-2) !} x^{2 k-2} y+\frac{1}{(2 k) !} x^{2 k}
$$

and

$$
Q_{k}(x, y)=\frac{1}{k !} x y^{k}+\frac{1}{(k-1) ! 3 !} x^{3} y^{k-1}+\ldots+\frac{1}{(2 k-1) !} x^{2 k-1} y+\frac{1}{(2 k+1) !} x^{2 k+1} .
$$

Then

$$
y^{2 k} P_{k}\left(1,-\frac{1}{2 y^{2}}\right)=\frac{1}{(2 k) !} H_{2 k}(y)
$$

and

$$
y^{2 k+1} Q_{k}\left(1,-\frac{1}{2 y^{2}}\right)=\frac{1}{(2 k+1) !} H_{2 k+1}(y) .
$$

Proof. Using Equation (5) we set

$$
f_{t}(y):=e^{t y-\frac{1}{2} t^{2}}=\sum_{n=0}^{\infty} H_{n}(y) \frac{t^{n}}{n !} .
$$

Now we split $f_{t}$ into its odd and even part $f_{t}^{e}$ and $f_{t}^{o}$, respectively, and get

$$
f_{t}^{e}(y)=\frac{1}{2}\left[f_{t}(y)+f_{t}(-y)\right] \quad \text { and } \quad f_{t}^{o}(y)=\frac{1}{2}\left[f_{t}(y)-f_{t}(-y)\right] .
$$


Furthermore,

$$
\begin{aligned}
& f_{t}^{e}(y)=\frac{1}{2}\left[e^{t y}+e^{-t y}\right] e^{-\frac{1}{2} t^{2}}=\sum_{n=0}^{\infty} H_{2 n}(y) \frac{t^{2 n}}{(2 n) !} \text { and } \\
& f_{t}^{o}(y)=\frac{1}{2}\left[e^{t y}-e^{-t y}\right] e^{-\frac{1}{2} t^{2}}=\sum_{n=0}^{\infty} H_{2 n+1}(y) \frac{t^{2 n+1}}{(2 n+1) !} .
\end{aligned}
$$

The product of the power series of $\cosh x$ and $e^{y}$ is absolute convergent. Therefore, we can arrange the summation in the following way:

$$
\begin{aligned}
(\cosh x) & e^{y} \\
= & \left(1+\frac{x^{2}}{2 !}+\frac{x^{4}}{4 !}+\frac{x^{6}}{6 !}+\ldots\right)\left(1+y+\frac{y^{2}}{2 !}+\frac{y^{3}}{3 !}+\frac{y^{4}}{4 !}+\frac{y^{5}}{5 !}+\frac{y^{6}}{6 !}+\ldots\right) \\
= & (y+1)+\left(\frac{1}{2 !} y^{2}+\frac{1}{2 !} x^{2} y+\frac{1}{4 !} x^{4}\right)+ \\
& \left(\frac{1}{3 !} y^{3}+\frac{1}{2 ! 2 !} x^{2} y^{2}+\frac{1}{4 !} x^{4} y+\frac{1}{6 !} x^{6}\right)+\ldots \\
= & \sum_{k=0}^{\infty} P_{k}(x, y)
\end{aligned}
$$

For $s \in \mathbb{R}$ it follows:

$$
\sum_{k=0}^{\infty} P_{k}\left(x, s x^{2}\right)=\sum_{k=0}^{\infty} x^{2 k} P_{k}(1, s)=(\cosh x) e^{s x^{2}}=\frac{1}{2}\left[e^{x}+e^{-x}\right] e^{s x^{2}} .
$$

By substituting $s=-\frac{1}{2 y^{2}}$ and $x=t y$, the above equation together with Equation (8) yields:

$$
\sum_{k=0}^{\infty}(t y)^{2 k} P_{k}\left(1,-\frac{1}{2 y^{2}}\right)=\sum_{k=0}^{\infty} H_{2 k}(y) \frac{t^{2 k}}{(2 k) !} .
$$

Now we get the formula

$$
y^{2 k} P_{k}\left(1,-\frac{1}{2 y^{2}}\right)=\frac{1}{(2 k) !} H_{2 k}(y)
$$

which proves the assertion.

In order to prove the second equation in Lemma 4.1 we consider the product of the power series of $\sinh x$ and $e^{y}$ and obtain

$$
\begin{aligned}
(\sinh & x) e^{y} \\
& =\left(x+\frac{x^{3}}{3 !}+\frac{x^{5}}{5 !}+\frac{x^{7}}{7 !}+\ldots\right)\left(1+y+\frac{y^{2}}{2 !}+\frac{y^{3}}{3 !}+\frac{y^{4}}{4 !}+\frac{y^{5}}{5 !}+\frac{y^{6}}{6 !}+\ldots\right) \\
= & \sum_{k=0}^{\infty} Q_{k}(x, y)
\end{aligned}
$$

By analogy with Equation (9) we obtain for $s \in \mathbb{R}$ :

$$
\sum_{k=0}^{\infty} Q_{k}\left(x, s x^{2}\right)=\sum_{k=0}^{\infty} x^{2 k+1} Q_{k}(1, s)=(\sinh x) e^{s x^{2}}=\frac{1}{2}\left[e^{x}-e^{-x}\right] e^{s x^{2}} .
$$


The substitutions $s=-\frac{1}{2 y^{2}}$ and $x=t y$ lead to

$$
\sum_{k=0}^{\infty}(t y)^{2 k+1} Q_{k}\left(1,-\frac{1}{2 y^{2}}\right)=\sum_{k=0}^{\infty} H_{2 k+1}(y) \frac{t^{2 k+1}}{(2 k+1) !}
$$

and thus

$$
y^{2 k+1} Q_{k}\left(1,-\frac{1}{2 y^{2}}\right)=\frac{1}{(2 k+1) !} H_{2 k+1}(y) \text {. }
$$

Using this lemma we can determine the roots of the following polynomials in one variable: For $c \in \mathbb{R}_{+}$let

$$
\begin{aligned}
g_{1, p}(t)= & \frac{1}{p !}(c t)^{p}+\frac{1}{(p-1) ! 2 !}(c t)^{p-1}+ \\
& \cdots \frac{1}{2 !(2 p-4) !}(c t)^{2}+\frac{1}{(2 p-2) !} c t+\frac{1}{(2 p) !}
\end{aligned}
$$

and

$$
\begin{aligned}
g_{2, p}(t)= & \frac{1}{p !}(c t)^{p}+\frac{1}{(p-1) ! 3 !}(c t)^{p-1}+ \\
& \cdots \frac{1}{2 !(2 p-3) !}(c t)^{2}+\frac{1}{(2 p-1) !} c t+\frac{1}{(2 p+1) !} .
\end{aligned}
$$

The roots of $g_{1, p}$ and $g_{2, p}$ are related to roots of the Hermite polynomials of order $2 p$ and $2 p+1$, because $g_{1, p}\left(\frac{x}{c}\right)=P_{p}(1, x)$ and $g_{2, p}\left(\frac{x}{c}\right)=Q_{p}(1, x)$. The roots of orthogonal polynomials are very well studied, and we can apply the following theorem about numbers of roots of Hermite polynomials (see e.g. [5]).

Theorem 4.2. The Hermite polynomial of order $n$ has exactly $n$ distinct real roots.

Theorem 4.2 and Lemma 4.1 give the following corollary:

Corollary 4.3. The polynomials $g_{1, p}$ and $g_{2, p}$ from Equation (10) and (11), respectively, have exactly $p$ negative roots. More precisely, the roots of $g_{1, p}$ are given by $-\frac{1}{2 c x_{i}^{2}}, i=1, \ldots, p$, where $x_{i}$ are the $p$ positive roots of $H_{2 p}$. The roots of $g_{2, p}$ are given by $-\frac{1}{2 c y_{i}^{2}}, i=1, \ldots, p$, where $y_{i}$ are the $p$ positive roots of $H_{2 p+1}$.

In order to prove the main part 2 of Theorem 1.1 we first consider the more general function $F_{l} \in \mathcal{C}^{\infty}\left(\mathbb{R}^{2}\right)$ for $l \in \mathbb{N}$ with

$$
F_{l}(x, y)=\sum_{\{k, i \mid k+2 i=l\}} \frac{1}{k ! i !} c^{i} x^{k} y^{i}+\sum_{\{k, i \mid k+2 i>l\}} \alpha_{i, k} x^{k} y^{i},
$$

where $c \in \mathbb{R}_{+}$and $\alpha_{i, k} \in \mathbb{R}$. The resolution of the singularity (0,0) into its Puiseux expansions is given by the following theorem:

Theorem 4.4. For $p \in \mathbb{N}$, let $x_{1}, \ldots, x_{p}$ be the $p$ distinct positive roots of the Hermite polynomial of order $2 p$ and $y_{1}, \ldots, y_{p}$ the $p$ distinct positive roots of the Hermite polynomial of order $2 p+1$. Furthermore, let $U$ be an open subset of $\mathbb{R}^{2}$ with $0 \in U$. Then there exist open neighborhoods $V, W \subset \mathbb{R}$ of $0, V \times W \subset U$, and $p$ convergent power series $h_{j}: V \rightarrow W$ and $n_{j} \in \mathbb{N}$ for $j=1, \ldots, p$ with

$$
h_{j}(x)=t_{j} x^{2}+\sum_{i=1}^{\infty} a_{i, j} x^{2+\frac{i}{n_{j}}}
$$


and $t_{j}<0$ for all $j=1, \ldots, p, t_{i} \neq t_{j}$, such that $F_{l}\left(x, h_{j}(x)\right)=0$.

We distinguish two cases.

1. Let $l=2 p$ and $t_{j}=-\frac{1}{2 c x_{j}^{2}}$. Then $\left\{\left(x, h_{j}(x)\right) \mid x \in V\right\}$ is exactly the set of solutions of $F_{l}(x, y)=0$ in $V \times W$.

2. Let $l=2 p+1$ and $t_{j}=-\frac{1}{2 c y_{j}^{2}}$. There exist $\rho \in \mathbb{Q}, \rho>0$, and a convergent power series $F_{1}(x, y)$, such that $F_{l}(x, y)=x^{\rho} F_{1}(x, y)$ and $\left\{\left(x, h_{j}(x)\right) \mid x \in V\right\}$ is exactly the set of solutions of $F_{1}(x, y)=0$ in $V \times W$.

Proof. In the following we will resolve the singular point $(0,0)$ of $F_{l}$ by applying the Newton Process. We consider the cases $l$ odd and $l$ even separately.

1. Let $l=2 p$ for $p \in \mathbb{N}$. For $\mu_{0}=2$ and $\nu=2 p$, we can split the function $F_{l}$ into the sum of a polynomial $\tilde{F}_{l}$ and a remainder $\tilde{H}_{l}$ (cf. Equation (7)). The approximation $\tilde{F}_{l}$ is given by

$$
\begin{aligned}
\tilde{F}_{l}(x, y)= & \frac{1}{p !}(c y)^{p}+\frac{1}{(p-1) ! 2 !} x^{2}(c y)^{p-1}+\ldots \\
& +\frac{1}{2 !(2 p-4) !} x^{2 p-4}(c y)^{2}+\frac{1}{(2 p-2) !} x^{2 p-2} c y+\frac{1}{(2 p) !} x^{2 p}
\end{aligned}
$$

and after substituting $y=t x^{2}$ we get $\tilde{F}_{l}(x, y)=x^{2 p} g(t)$ with

$$
\begin{aligned}
g(t)= & \frac{1}{p !}(c t)^{p}+\frac{1}{(p-1) ! 2 !}(c t)^{p-1}+\cdots \\
& \frac{1}{2 !(2 p-4) !}(c t)^{2}+\frac{1}{(2 p-2) !} c t+\frac{1}{(2 p) !}
\end{aligned}
$$

Since $g$ is the polynomial $g_{1, p}$ from Equation (10), we know from Corollary 4.3 that $g$ has $p$ negative roots $t_{1}, \ldots, t_{p}$ with $t_{j}=-\frac{1}{2 c x_{i}^{2}}$, where $x_{i}$ are the positive roots of $H_{2 p}$. Therefore, the zero set of $F_{l}$ is parametrized by the Puiseux expansions

$$
y_{j}(x)=\sum_{i=2 n_{j}} a_{i, j}\left(x^{\frac{1}{n_{j}}}\right)^{i}
$$

with $n_{j} \in \mathbb{N}$ and $a_{2 n_{j}, j}=t_{j}$; this means $y_{j}(x)=t_{j} x^{2}+O\left(x^{2+\frac{1}{n_{j}}}\right)$.

2. Let $l=2 p+1$ for $p \in \mathbb{N}$. If we set $\mu_{0}=2$ and $\nu=2 p+1$, then $\frac{l}{2}=\frac{2 p+1}{2} \notin \mathbb{N}$, and therefore we cannot start the Newton process.

a) Assume that $\alpha_{0, i}=0$ for all $i \in \mathbb{N}$. Then

$$
F_{l}(x, y)=x F_{l}^{*}(x, y) \quad \text { with } \quad F_{l}^{*}(x, y)=\sum_{k+2 i \geq 2 p} \gamma_{k+1, i} x^{k} y^{i}
$$

where $\gamma_{k, i}=\frac{1}{k ! i !} \alpha_{k, i}$, and the condition $\frac{\mu_{0}}{\nu} \in \mathbb{N}$ is valid for $\mu_{0}=2$ and $\nu=2 p$. The first approximation $\tilde{F}_{l}^{*}$ is presented as

$$
\begin{aligned}
\tilde{F}_{l}^{*}(x, y)= & \frac{1}{p !}(c y)^{p}+\frac{1}{(p-1) ! 3 !} x^{2}(c y)^{p-1}+\ldots \\
& +\frac{1}{2 !(2 p-3) !} x^{2 p-4}(c y)^{2}+\frac{1}{(2 p-1) !} x^{2 p-2} c y+\frac{1}{(2 p+1) !} x^{2 p} .
\end{aligned}
$$


The solutions of $F_{l}^{*}(x, y)=0$ are determined in the same way as in 1$)$, except that the $t_{j}$ are the roots of the polynomial

$$
\begin{aligned}
g(t)= & \frac{1}{p !}(c t)^{p}+\frac{1}{(p-1) ! 3 !}(c t)^{p-1}+\ldots \\
& \frac{1}{2 !(2 p-3) !}(c t)^{2}+\frac{1}{(2 p-1) !} c t+\frac{1}{(2 p+1) !} .
\end{aligned}
$$

Since $g$ is the polynomial $g_{2, p}$ from Equation (11), we know from Corollary 4.3 that $g$ has $p$ negative roots $t_{1}, \ldots, t_{p}$ with $t_{j}=-\frac{1}{2 c y_{i}^{2}}$, where $x_{i}$ are the positive roots of $H_{2 p+1}$.

b) There exists $m \in \mathbb{N}$, such that $\alpha_{0, m} \neq 0$ and $\alpha_{0, i}=0$ for all $i<m$. For $\gamma_{k, i}=\frac{1}{k ! i !} \alpha_{k, i}$ we get

$$
F_{l}(x, y)=\sum_{k+\mu_{0} i \geq \nu} \gamma_{k, i} x^{k} y^{i} \quad \text { with } \quad \mu_{0}=\frac{1}{m-p} \quad \text { and } \quad \nu=\frac{m}{m-p} .
$$

The substitutions $y=x^{\frac{1}{m-p}} y_{1}$ and $x_{1}=x^{\frac{1}{m-p}}$ yield

$$
\begin{aligned}
F_{l}(x, y)= & F_{l}\left(x, x^{\frac{1}{m-p}} y_{1}\right)=F_{l}\left(x_{1}^{m-p}, x_{1} y_{1}\right) \\
= & \gamma_{1, p} x_{1}^{m-p} x_{1}^{p} y_{1}^{p}+\ldots+\gamma_{2 p-1,1} x_{1}^{(2 p-1)(m-p)} x_{1} y_{1} \\
& +\gamma_{2 p+1,0} x_{1}^{(2 p+1)(m-p)}+\gamma_{0, m} x_{1}^{m} y_{1}^{m}+\ldots \\
= & x_{1}^{m}\left[\gamma_{1, p} y_{1}^{p}+\gamma_{3, p-1} x_{1}^{2(m-p)-1} y_{1}^{p-1}+\gamma_{5, p-2} x_{1}^{2[2(m-p)-1]} y_{1}^{p-2}+\ldots\right. \\
& \left.\ldots+\gamma_{2 p-1,1} x_{1}^{(p-1)[2(p-m)-1]} y_{1}+\gamma_{2 p+1,0} x_{1}^{p[2(p-m)-1]}+\gamma_{0, m} y_{1}^{m}+\ldots\right] \\
= & x_{1}^{m} F_{l}^{*}\left(x_{1}, y_{1}\right) .
\end{aligned}
$$

The power series $F_{l}^{*}$ satisfies the assumptions of the Newton process $\left(\mu_{1}=\right.$ $\left.2(m-p)-1, \nu_{1}=p[2(m-p)-1]\right)$, and for the first approximation $\tilde{F}_{l}^{*}$ we get:

$$
\begin{aligned}
\tilde{F}_{l}^{*}\left(x_{1}, y_{1}\right)= & \gamma_{1, p}\left(c y_{1}\right)^{p}+\gamma_{3, p-1} x_{1}^{\mu_{1}}\left(c y_{1}\right)^{p-1}+\ldots \\
& +\gamma_{2 p-1,1} x_{1}^{(p-1) \mu_{1}} c y_{1}+\gamma_{2 p+1,0} x_{1}^{p \mu_{1}} .
\end{aligned}
$$

If we substitute $y_{1}=t x_{1}^{\mu_{1}}$, we obtain $\tilde{F}_{l}^{*}\left(x_{1}, y_{1}\right)=x_{1}^{p \mu_{1}} g(t)$, where $g$ is the polynomial $g_{2, p}$ from Equation (11). Therefore, the zero set of $F_{l}^{*}$ is parametrized by the Puiseux expansions

$$
y_{1, j}(x)=\sum_{i=n_{j} \mu_{1}} a_{i, j}\left(x_{1}^{\frac{1}{n_{j}}}\right)^{i},
$$

where $n_{j} \in \mathbb{N}$ and $a_{2 n_{j}, j}=t_{j}$. In terms of $x$ and $y$ the solutions of $F_{l}^{*}(x, y)=0$ are given by

$$
\begin{aligned}
y_{j}(x) & =\sum_{i=n_{j} \mu_{1}} a_{i, j} x^{\frac{n_{j}+i}{n_{j}(m-p)}} \\
& =t_{j} x^{2}+O\left(x^{2+\frac{1}{n_{j}(m-p)}}\right) .
\end{aligned}
$$


We are now able to prove part 2 of the Main Theorem. Let $\left(b_{0}, a_{0}\right)$ be a singular zero of $T_{\theta}[f]$ such that

$$
\frac{\partial^{m} T_{\theta}[f]}{\partial b^{m}}\left(b_{0}, a_{0}\right)=0 \quad \text { for all } m<n \quad \text { and } \frac{\partial^{n} T_{\theta}[f]}{\partial b^{n}}\left(b_{0}, a_{0}\right) \neq 0 .
$$

Using the information about the partial derivatives of $T_{\theta}[f]$ from Corollary 2.2 we reduce the Taylor exansion of $T_{\theta}[f]$ in $\left(b_{0}, a_{0}\right)$ to

$$
\begin{aligned}
T_{\theta}[f](b, a)= & \sum_{k+2 i=n}^{\infty} \frac{1}{k ! i !}\left(a_{0}\right)^{i}\left(b-b_{0}\right)^{k}\left(a-a_{0}\right)^{i} \frac{\partial^{n} T_{\theta}[f]}{\partial b^{n}}\left(b_{0}, a_{0}\right)+ \\
& \sum_{k+2 i>n}^{\infty} \frac{1}{k ! i !}\left(b-b_{0}\right)^{k}\left(a-a_{0}\right)^{i} \frac{\partial^{k+i} T_{\theta}[f]}{\partial a^{i} \partial b^{k}}\left(b_{0}, a_{0}\right) .
\end{aligned}
$$

The Taylor expansion has exactly the form $F_{l}$ for $l=n$. Therefore, part 2 of Theorem 1.1 follows from Theorem 4.4.

\section{Further Relations to Hermite polynomials}

First, we give an example of a class of functions $f: \mathbb{R} \rightarrow \mathbb{R}$, such that the zero set of $T_{\theta}[f]$ has a singularity of type (a) of (b) in Theorem 1.1.

Example 5.1. Let $\left(b_{0}, a_{0}\right) \in \mathbb{R} \times \mathbb{R}_{+}, n \in \mathbb{N}$ and

$$
f\left(a_{0} x+b_{0}\right)=\sum_{i=n}^{\infty} c_{i} H_{i}(x),
$$

a convergent Hermite series with $c_{i} \in \mathbb{R}, c_{n} \neq 0$. From Equation (6) we get

$$
\frac{\partial^{m} T_{\theta}[f]}{\partial b^{m}}\left(b_{0}, a_{0}\right)=\frac{1}{a_{0}^{m}} \int\left[c_{n} H_{n}(x)+\sum_{i=n+1}^{\infty} c_{i} H_{i}(x)\right] H_{m}(x) e^{-\frac{1}{2} x^{2}} d x=0
$$

for all $m<n$ and

$\frac{\partial^{n} T_{\theta}[f]}{\partial b^{n}}\left(b_{0}, a_{0}\right)=\frac{1}{a_{0}^{n}} \int\left[c_{n} H_{n}(x)+\sum_{i=n+1}^{\infty} c_{i} H_{i}(x)\right] H_{n}(x) e^{-\frac{1}{2} x^{2}} d x=\frac{1}{a_{0}^{n}} c_{n} n ! \sqrt{2 \pi}$.

Thus, for each $n \in \mathbb{N}$ there exist functions $f \in L_{\theta}^{2}(\mathbb{R})$ with the desired type of singularity.

Conversely, we can ask whether a given bifurcation point of type (a) or (b) in Theorem 1.1 determines the underlying function $f$. To answer this question we use the following theorem (see e.g. [8]):

Theorem 5.2. Let $f \in L_{\theta}^{2}(\mathbb{R})$ be continuous with a piecewise continuous derivative $f^{\prime} \in L_{\theta}^{2}(\mathbb{R})$. Then $f(x)=\sum_{n=0}^{\infty} c_{n} H_{n}(x)$ for all $x \in \mathbb{R}$, where $c_{n}=$ $\frac{1}{n ! \sqrt{2 \pi}} \int_{-\infty}^{\infty} f(x) H_{n}(x) e^{-\frac{1}{2} x^{2}} d x$. Moreover, the sequence $f_{n}(x)=\sum_{k=0}^{n} c_{k} H_{k}(x)$ converges uniformly to $f$ on each interval $\left[x_{1}, x_{2}\right] \subset \mathbb{R}$.

From this theorem we derive the following lemma:

Lemma 5.3. Let $f \in L_{\theta}^{2}(\mathbb{R})$ be continuous and $f^{\prime} \in L_{\theta}^{2}(\mathbb{R})$ be piecewise continuous. Then $\frac{\partial^{m} T_{\theta}[f]}{\partial^{m} b}(b, a)=0$ for $m<n, \frac{\partial^{n} T_{\theta}[f]}{\partial^{n} b}(b, a) \neq 0$ iff $f(a x+b)=\sum_{i=n}^{\infty} c_{i}^{a, b} H_{i}(x)$, where $c_{i}^{a, b} \in \mathbb{R}, c_{n}^{a, b} \neq 0$. 


\section{ACKNOWLEDGMENT}

The author would like to thank Fritz von Haeseler, Heinz-Otto Peitgen and Raymond O. Wells, Jr. for many discussions on various parts of this paper. Part of this work was done while the author was working at the Center for Complex Systems and Visualization, University of Bremen. During that time she was a member of the Forschergruppe Dynamische Systeme, supported by the DFG.

\section{REFERENCES}

[1] Anh, V.; Shi, J.Y.; Tsui, H.T. Scaling Theorems for Zero Crossings of Bandlimited Signals, IEEE Trans. Patt. Anal. Mach. Intell. Vol. 18, No. 3, 1996, pp. 309-320.

[2] Babaud, J. et al. Uniqueness of the Gaussian Kernel for Scale-Space Filtering, IEEE Trans. Patt. Anal. Mach. Intell. Vol.PAMI-8, No.1, 1986, pp. 26-33.

[3] Berkner, K. Eine Charakterisierung der Nullstellen-Mengen von Gauß-Faltungen und eine daraus resultierende lokale Beschreibung von Kanten in Bildern, Dissertation, Universität Bremen, 1996.

[4] Brieskorn,E.; Knörrer, H. Plane Algebraic Curves, Birkhäuser Verlag, Basel, 1986. MR 88a:14001

[5] Chihara, T. S. An Introduction to Orthogonal Polynomials, Gordon and Breach, Science Publishers, New York, 1978. MR 58:1979

[6] Freud, G. Orthogonale Polynome Birkhäuser Verlag, Basel, 1969. MR 58:1982

[7] Johansen, P. et al. Representing Signals by Their Toppoints in Scale Space, Proc. 8th Int. Conf. Pattern Recogn., Vol. 1, 1986, pp. 215-217.

[8] Nikiforov, A.F.; Uvarov, V.B. Special Functions of Mathematical Physics, Birkhäuser Verlag, Basel, 1988. MR 89h:33001

[9] Wu, L.; Xie, Z. Scaling Theorems for Zero Crossings, IEEE Trans. Patt. Anal. Mach. Intell. Vol. 12, No.1, 1990, pp. 46-54.

[10] Yuille, A.L.; Poggio, T.A. Scaling Theorems for Zero Crossings, IEEE Trans. Patt. Anal. Mach. Intell. Vol. 8, No.1, 1986, pp. 15-25.

Department of Mathematics, Rice University, Houston, Texas 77251-1892

E-mail address: berkner@cml.rice.edu 